\title{
Influência da idade do hospedeiro e da aprendizagem no comportamento quimiotáxico e no parasitismo de Trichogramma pretiosum
}

\author{
Camila C. Vargas' ', Luiza R. Redaelli', Josué Sant'Ana', Rosana M. Morais² \& Priscila Padilha'
}

1. Programa de Pós-Graduação em Fitotecnia, Faculdade de Agronomia, Universidade Federal do Rio Grande do Sul, Av. Bento Gonçalves, 7712, 91540-000
Porto Alegre, RS, Brasil. (teccamila@gmail.com; luredael@ufrgs.br; josue.santana@ufrgs.br; prici.padilha2@gmail.com)
2. Fundação Estadual de Pesquisa Agropecuária, Centro de Pesquisa em Florestas, Caixa Postal 346, 97001-970 Santa Maria, RS, Brasil. (entomorais@gmail.com)

Recebido 4 maio 2016

Aceito 8 fevereiro 2017

DOI: $10.1590 / 1678-4766 e 2017015$

\begin{abstract}
Influence of host age and learning on chemotactic behavior and parasitism of Trichogramma pretiosum. Trichogramma pretiosum Riley, 1879 reared on Ephestia kuehniella Zeller, 1879 eggs, have been used in biological control of Spodoptera frugiperda (J. E. Smith, 1797). However, host age and experience just after emergence might have some influence on parasitism and parasitoid behavior. This study evaluated the influence of $S$. frugiperda host age and egg odor experience, on chemotactic behavior and parasitism of T. pretiosum. Spodoptera frugiperda eggs with 24,48 and 72 hours were exposed to T. pretiosum females. Parasitoid time experience, in the same host, was evaluated within 1, 3, 4, 5, 6 and 24 hours, as well as, its host exposed time (1, 2, 3 and 24 hours). Chemotactic responses of $T$. pretiosum (experienced and inexperienced females) to $S$. frugiperda egg extracts were recorded in Y-tube olfactometer. We also observe parasitism rates (choice tests) in insects with and without experience. The parasitism average was higher in S. frugiperda eggs with 24 hours. Inexperienced females and those experienced for 1, 3 and 4 hours, parasitized less S. frugiperda eggs compared to those for 5, 6 and 24 hours. There was no difference in parasitism from females exposed for 2, 3 and 24 hours when compared to those exposed for one hour. Experienced females were more attracted to $S$. frugiperda eggs odor than to control (hexano). Parasitism percentage, by inexperienced parasitoids, was greater in E. kuehniella eggs (original host) than in S. frugiperda, however it was not observed in experienced females.
\end{abstract}

KEYWORDS: Biological control, egg parasitoid, eggs extract.

RESUMO. Trichogramma pretiosum Riley, 1879 mantido em ovos de Ephestia kuehniella Zeller, 1879, tem sido utilizado no controle biológico de Spodoptera frugiperda (J. E. Smith, 1797). No entanto, fatores como idade do hospedeiro e experiência prévia, podem influenciar o parasitismo e o comportamento do parasitoide. Esse estudo avaliou a influência da idade dos ovos e da experiência em ovos e extrato de ovos de $S$. frugiperda no comportamento quimiotáxico e no parasitismo de $T$. pretiosum. Ovos de $S$. frugiperda com 24,48 e 72 horas, foram expostos a fêmeas de $T$. pretiosum. Também foi avaliado o tempo de experiência do parasitoide, no mesmo hospedeiro, por 1, 3, 4, 5, 6 e 24 horas, assim como, seu tempo de exposição (1, 2, 3 e 24 horas). As respostas quimiotáxicas de T. pretiosum (experiente e não experiente) em extrato de ovos de $S$. frugiperda foram observadas em olfatômetro tipo Y. As taxas de parasitismo foram registradas em teste de escolha, com insetos experientes e inexperientes com ovos e extrato de ovos de $S$. frugiperda. O parasitismo em $S$. frugiperda foi significativamente maior em ovos com 24 horas de idade. Fêmeas inexperientes e experientes por 1, 3 e 4 horas, apresentaram uma menor taxa de parasitismo, quando comparadas a fêmeas expostas por 5,6 e 24 horas. Não houve diferença de parasitismo em fêmeas expostas por 2, 3 e 24 horas, comparadas às expostas por uma hora. Fêmeas experientes foram mais atraídas ao extrato de ovos de $S$. frugiperda, do que ao controle (hexano). A porcentagem de parasitismo, em fêmeas inexperientes, foi maior em ovos de E. kuehniella (hospedeiro de origem), no entanto, o mesmo resultado não foi observado em fêmeas experientes.

PALAVRAS-CHAVE: Controle biológico, parasitoide de ovos, extrato de ovos.

Parasitoides de ovos Trichogramma (Hymenoptera: Trichogrammatidae) são utilizados em programas de controle biológico (AlmeIDA et al., 2010; Davies et al., 2011), com destaque para o controle de Spodoptera frugiperda (J. E. Smith, 1797) (Lepidoptera: Noctuidae) com Trichogramma pretiosum Riley, 1869 (Beserra \& PARRa, 2005; Figueiredo et al. 2015; Querino et al., 2016). O sucesso da espécie como agente de controle biológico, depende do conhecimento de suas características bioecológicas relacionadas à interação com o hospedeiro (SiqueIra et al., 2011), como a sua habilidade de localizar e parasitar a praga-alvo (GAZIT et al., 1996).
A localização do hospedeiro está relacionada à presença de aleloquímicos como os cairomônios que podem atuar a médias e curtas distâncias ou mesmo por contato. Para que ocorra o parasitismo o inseto avalia o hospedeiro através, principalmente, dos voláteis e toque (tamborilamento) os quais poderão fornecer informações sobre a idade do ovo (VINSON, 1998). A preferência de fêmeas, por parasitar ovos com menor desenvolvimento embrionário, foi observada em Trichogramma exiguum Pinto \& Platner, 1978 em ovos Mocis latipes (Grennée, 1852) (Lepidoptera: Noctuidae) (Zuim et al., 2013) e Trichogramma ostrinidae Pang \& Chen, 1974 em ovos de Chilo suppressalis Walker, 1863 (Lepidoptera: 
Pyralidae) (Zhang et al., 2014).

Outro fator importante ao parasitismo refere-se ao hospedeiro de origem (SuJII et al., 2002). A criação massal de T. pretiosum geralmente é conduzida em ovos de Ephestia kuehniella (Zeller, 1879) (PARRA, 1997). No entanto, a multiplicação dos parasitoides em um mesmo hospedeiro por longo período, pode afetar o seu comportamento de busca (CARNEIRO et al., 2006). GoulART et al. (2011), por exemplo, verificaram que o parasitismo de $T$. pretiosum oriundo de Anticarsia gemmatalis (Hübner, 1818) (Lepidoptera: Noctuidae) foi maior nessa espécie do que em $S$. frugiperda e E. kuehniella. O mesmo já havia sido observado por VolPE et al. (2006) que registraram maior número de ovos parasitados por indivíduos desta espécie, em seu hospedeiro de origem, E. kuehniella, comparado com Plutella xylostella (Linnaeus, 1758) (Lepidoptera: Plutellidae) e $S$. frugiperda. De fato, HoPKIns (1917) já havia constatado que parasitoides preferem ovipositar na mesma espécie na qual se desenvolveram, sendo que as informações obtidas na fase imatura também poderiam ser transmitidas ao adulto através do legado químico de seu hospedeiro.

A aprendizagem em Trichogramma também pode ocorrer na fase adulta (BARRON, 2001). Segundo o autor, este fato está relacionado ao novo princípio de Hopkins, o qual enfatiza a importância da experiência na fase adulta dos insetos, sobre a escolha do hospedeiro. NURINDAH et al. (1999) observaram que o tempo de reconhecimento e oviposição de Trichogramma australicum Girault, 1912 diminuiu após a primeira inserção do ovipositor em ovos de Helicoverpa armigera Hübner, 1809 (Lepidoptera: Noctuidae), corroborando este princípio.

O conhecimento a respeito da aprendizagem na fase adulta de $T$. pretiosum pode permitir a manipulação do comportamento quimiotáxico desta espécie de forma a incrementar a sua busca por hospedeiros, resultando em aumento no parasitismo. Neste contexto, este trabalho objetivou estudar a influência da idade do hospedeiro e da aprendizagem no comportamento quimiotáxico e no parasitismo de $T$. pretiosum.

\section{MATERIAL E MÉTODOS}

Os bioensaios foram realizados no Laboratório de Biologia, Ecologia e Controle Biológico de Insetos (BIOECOLAB) e no Laboratório de Etologia e Ecologia Química de Insetos (LEEQI) da UFRGS, em ambiente climatizado $\left(25 \pm 2{ }^{\circ} \mathrm{C}, 65 \pm 10 \%\right.$ UR, fotofase de 12 horas $)$. A linhagem de $T$. pretiosum utilizada foi proveniente de espécimes coletados em ovos de Helicoverpa zea (Boddie, 1850) (Lepidoptera: Noctuidae), em lavoura de milho, situada na Estação Experimental da FEPAGRO/Floresta (2941'24'S; 5348'42”W), em Santa Maria, Rio Grande do Sul. Estes foram multiplicados e mantidos em ovos de E. kuehniella. As criações de $T$. pretiosum e de $S$. frugiperda seguiram as metodologias de PARRA (1997, 2001), respectivamente. Todas as fêmeas de $T$. pretiosum utilizadas nos bioensaios foram criadas em ovos de E. kuehniella. Para os testes de experiência, exposição, resposta quimiotáxica e parasitismo, foram utilizados ovos de $S$. frugiperda de até 24 horas de idade.

Avaliação da idade dos ovos. Ovos de $S$. frugiperda com idades de 24, 48 e 72 horas, foram agrupados $(n=20)$ por idade e fixados em cartelas $(1 \mathrm{x} 1 \mathrm{~cm})$ de papel sulfite branco $\left(75 \mathrm{~g} / \mathrm{m}^{2}\right)$ utilizado como substrato de oviposição do hospedeiro. Cada cartela foi disposta em um tubo de vidro $(0,5 \times 3 \mathrm{~cm})$, onde foi liberada uma fêmea de T. pretiosum (< 36 horas de idade) previamente pareada e exposta a ovos de S. frugiperda por 24 horas. Após este período de experiência, cada fêmea foi mantida por mais 24 horas com uma das posturas de cada uma das idades. Foram avaliados o número médio de ovos parasitados, o sucesso de parasitismo (número de ovos com registro de orifício de saída e emergência de adulto), a duração do desenvolvimento e a razão sexual (n $=15)$.

Avaliação do tempo de experiência. Fêmeas de $T$. pretiosum não experientes e experientes, com idade de até 24 horas foram avaliadas. Para aquisição de experiência, cada fêmea foi exposta a uma cartela com 20 ovos de $S$. frugiperda, preparada como descrito no bioensaio anterior, em tubos de vidro (0,5 x $3 \mathrm{~cm})$, por 1, 3, 4, 5, 6 e 24 horas. Imediatamente após a exposição, as fêmeas experientes receberam uma nova cartela com ovos de $S$. frugiperda $(\mathrm{n}=$ 20), em tubo de vidro $(0,5 \times 3 \mathrm{~cm})$, com a qual permaneceram por 24 horas. As fêmeas não experientes de mesma idade receberam cartelas semelhantes com as quais foram mantidas pelo mesmo tempo. Foram avaliados o número médio de ovos parasitados e o sucesso de parasitismo $(n=15)$.

Avaliação do tempo de exposição. Fêmeas de $T$. pretiosum com até 24 horas de idade, foram mantidas em experiência, por 5 horas com uma cartela contendo 20 ovos de $S$. frugiperda, em tubos de vidro $(0,5 \times 3 \mathrm{~cm})$. Transcorrido esse período, as fêmeas foram retiradas e individualmente expostas à outra cartela com 20 ovos deste hospedeiro, por 1, 2, 3 e 24 horas. Os ovos foram mantidos em câmara climatizada até a emergência dos parasitoides ou eclosão de lagartas. Foram avaliados o número médio de ovos parasitados e o sucesso de parasitismo $(n=15)$.

Aprendizagem. Resposta quimiotáxica. Ovos de $S$. frugiperda com até 24 horas de idade foram destacados do substrato de oviposição e separados das escamas, com auxílio de pincel fino ( $\left.{ }^{\circ} 000\right)$, utilizando luvas de látex sem pó bioabsorvível. Um grama de ovos foi mantido em solução com $4 \mathrm{~mL}$ de hexano (solvente) (P.A., grau HPLC ou equivalente/Sigma Aldrich) por cinco minutos. Após, o solvente foi coletado e armazenado em vial a $-4^{\circ} \mathrm{C}$. Fêmeas de $T$. pretiosum com até 24 horas de idade, foram alocadas em tubos de vidro $(0,5 \times 3 \mathrm{~cm})$, revestidos com papel filtro ( 1 x $3,7 \mathrm{~cm})$, contendo $10 \mu \mathrm{L}$ de extrato, onde permaneceram por 5 horas para aquisição de experiência. Fêmeas sem experiência foram mantidas nas mesmas condições, porém em contato com $10 \mu \mathrm{L}$ de hexano (controle).

As respostas quimiotáxicas foram avaliadas em fêmeas experientes e inexperientes, em olfatômetro de vidro de dupla escolha, com diâmetro de 1,4 cm, arena inicial de 
$16 \mathrm{~cm}$, bifurcada em dois "braços" de $19 \mathrm{~cm}$ cada, sob luz fluorescente ( 60 W, luminância 290 lux). Na extremidade de um dos braços foi colocado um papel filtro $\left(80 \mathrm{~g} / \mathrm{m}^{2}\right), 1 \times 3,7$ $\mathrm{cm}$, dobrado em forma de gaita, contendo $10 \mu \mathrm{L}$ do extrato de ovos, na outra, um papel com o mesmo volume do solvente. Um fluxo de ar, previamente filtrado com carvão ativo, foi conduzido para dentro do sistema com auxílio de uma bomba a vácuo conectada a um fluxímetro e um umidificador, a uma taxa de $0,25 \mathrm{~L} / \mathrm{min}$. O olfatômetro era invertido no sentido horizontal (rotação $180^{\circ}$ ) a cada três repetições e, a cada seis, lavado com sabão neutro e hexano e seco em estufa de esterilização a $150{ }^{\circ} \mathrm{C}$. Após este procedimento, eram renovadas as substâncias testadas. Foram realizadas, no mínimo, 44 repetições por tratamento. As respostas foram consideradas positivas quando os parasitoides alcançavam a fonte de odor e percorriam, pelo menos, $4 \mathrm{~cm}$ dentro dos braços e permaneciam nesta área por, no mínimo, um minuto. Consideraram-se não responsivos os insetos que não se movimentaram nos primeiros cinco minutos ou que não alcançaram os braços do olfatômetro em dez minutos.

Aprendizagem. Parasitismo. Fêmeas de T. pretiosum com até 24 horas de idade foram, individualmente, expostas em tubos de vidro $(0,5 \times 3 \mathrm{~cm})$, por 5 horas, a três tratamentos: a) cartela contendo 20 ovos de $S$. frugiperda; b) papel filtro com $10 \mu \mathrm{L}$ de extrato de ovos de S. frugiperda e c) papel filtro com $10 \mu \mathrm{L}$ de hexano. Imediatamente após o período de experiência, as fêmeas foram colocadas em tubos de vidro, por 2 horas, com 20 ovos de E. kuehniella e 20 de $S$. frugiperda, dispostos cada grupo nas extremidades de uma mesma cartela $(3 \times 2 \mathrm{~cm})$ de papel sulfite branco $(75 \mathrm{~g} /$ $\mathrm{m}^{2}$ ), separados por, aproximadamente, $1 \mathrm{~cm}$. Após este procedimento, ovos de mesma cartela foram separados por espécie e armazenados, até a emergência dos parasitoides ou eclosão das larvas $(n=30)$.

Análise estatística. O número médio de ovos parasitados, a duração do desenvolvimento e a razão sexual foram testados quanto à normalidade por Shapiro-Wilks. Por não apresentarem distribuição normal foram analisados pelo teste de Kruskal-Wallis, como comparação das médias pelo teste de Dunn. Os percentuais de respostas quimiotáxicas e de parasitismo foram comparados pelo teste de Qui-quadrado. As análises foram efetuadas utilizando-se o programa Bioestat $5.0^{\circledR}$ (AYRES et al., 2007), ao nível de significância de 5\%.

\section{RESULTADOS}

Idade dos ovos. O número médio de ovos parasitados foi significativamente maior em ovos de 24 horas de idade, em relação aos de 48 e 72, os quais também diferiram entre si (H $=28.519 ; \mathrm{gl}=2 ; \mathrm{p}=0,0001)$. O tempo de desenvolvimento de $T$. pretiosum foi semelhante entre os ovos de 24 e 48 horas, e maior nos de $72(\mathrm{H}=34.381 ; \mathrm{gl}=2 ; \mathrm{p}=0,0001)$. O sucesso de parasitismo foi de $100 \%$ para os três tratamentos. A razão sexual foi semelhante entre eles $(\mathrm{H}=0,3205$; $\mathrm{gl}=$ $2 ; \mathrm{p}=0,8519)($ Tab. I).

Tempo de experiência. Fêmeas sem experiência $(10,7$ $\pm 1,35)$ e experientes por $1(10,3 \pm 0,45), 3(14,1 \pm 0,66) \mathrm{e}$
Tab. I. Média de ovos parasitados ( \pm EP), duração média do desenvolvimento (dias), razão sexual da prole, número de parasitoides emergidos por ovo de Trichogramma pretiosum Riley, 1879 expostos a ovos de Spodoptera frugiperda (J. E. Smith, 1797), de diferentes idades $\left(25 \pm{ }^{\circ} \mathrm{C}, 65 \pm 10 \%\right.$ UR, fotofase 12 horas $)(n=15)$.

\begin{tabular}{cccc}
\hline $\begin{array}{c}\text { Idades } \\
(\mathrm{h})\end{array}$ & $\begin{array}{c}\text { Ovos } \\
\text { parasitados }^{1}\end{array}$ & $\begin{array}{c}\text { Desenvolvimento } \\
(\text { dias })^{1}\end{array}$ & $\begin{array}{c}\text { Razão } \\
\text { sexual }^{2}\end{array}$ \\
\hline 24 & $18,6 \pm 0,38 \mathrm{a}$ & $8,6 \mathrm{a}$ & 0,46 \\
48 & $16,6 \pm 0,36 \mathrm{~b}$ & $9,1 \mathrm{a}$ & 0,50 \\
72 & $14,4 \pm 0,36 \mathrm{c}$ & $10,0 \mathrm{~b}$ & 0,49 \\
\hline
\end{tabular}

${ }^{1}$ Médias seguidas de mesma letra, nas colunas, não se diferem entre si pelo teste de Kruskal-Wallis, a 5\% de significância.

${ }^{2} \mathrm{~ns}=$ não significativa entre si pelo teste de Kruskal-Wallis, a 5\% de significância.

$4(14,1 \pm 0,47)$ horas a ovos de $S$. frugiperda parasitaram $31,17 \%$ a menos que fêmeas experientes por $5(16,3 \pm 1,43), 6$ $(16,1 \pm 1,48)$ e $24(18,6 \pm 1,50)$ horas, as quais não diferiram entre si $(H=62,014 ; g l=6 ; p=0,0001)$. O sucesso de parasitismo foi de $100 \%$ em todos os tratamentos.

Tempo de exposição. Não houve diferença na média de ovos parasitados entre fêmeas expostas por $2(16,1 \pm$ $0,66), 3(16,3 \pm 0,89)$ e 24 horas $(18,2 \pm 0,36)$, sendo estas maiores que as das expostas por uma hora $(11,7 \pm 0,84)(\mathrm{H}$ $=25,1565 ; \mathrm{gl}=3 ; \mathrm{p}=0,0001)$. O sucesso de parasitismo foi de $100 \%$ em todos os tratamentos.

Aprendizagem - Resposta quimiotáxica. Fêmeas experientes ao extrato de ovos de $S$. frugiperda foram significativamente mais atraídas a este odor do que ao hexano $\left(\chi^{2} \mathrm{cal}=47,8123 ; \mathrm{gl}=1 ; \mathrm{p} \leq 0,0001\right)$. As inexperientes responderam de forma igual aos dois tratamentos $\left(\chi^{2} \mathrm{cal}=\right.$ $0,4244 ; \mathrm{gl}=1 ; \mathrm{p}=0,5148)$ (Fig. 1).

Aprendizagem - Parasitismo. Fêmeas de T. pretiosum sem experiência e oriundas de E. kuehniella, apresentaram preferência por seu hospedeiro de origem $\left(\chi^{2} \mathrm{cal}=252.4024\right.$; $\mathrm{gl}=1 ; \mathrm{p} \leq 0,0001)$. Entretanto quando avaliadas fêmeas oriundas de E. kuehniella, com prévia experiência em ovos $\left(\chi^{2} \mathrm{cal}=3.4218 ; \mathrm{gl}=1 ; \mathrm{p} \leq 0,0643\right)$ ou extrato de ovos de $S$. frugiperda, estas não apresentaram diferença significativa na escolha do hospedeiro $\left(\chi^{2} \mathrm{cal}=252.4024 ; \mathrm{gl}=1 ; \mathrm{p} \leq\right.$ 0,0001) (Fig. 2).

\section{DISCUSSÃO}

A idade do ovo hospedeiro interferiu na taxa de parasitismo e na duração do desenvolvimento de $T$. pretiosum. Segundo VINSON (1976), este é um dos fatores mais importantes na interação hospedeiro-parasitoide. A preferência por ovos mais novos está relacionada com características internas e externas destes e pode variar entre espécies ou linhagens de Trichogramma (ScHMIDT \& SMITH, 1988). Embriões com maior tempo de desenvolvimento esgotam os nutrientes armazenados no ovo, apresentando baixa qualidade para o parasitoide, e sua larva não é capaz de digerir a cutícula do hospedeiro e absorver os nutrientes (Strand \& Vinson, 1986). Além disso, com o avanço do desenvolvimento embrionário, o córion torna-se endurecido e limitante para a penetração do ovipositor (PAK et al., 1986). 


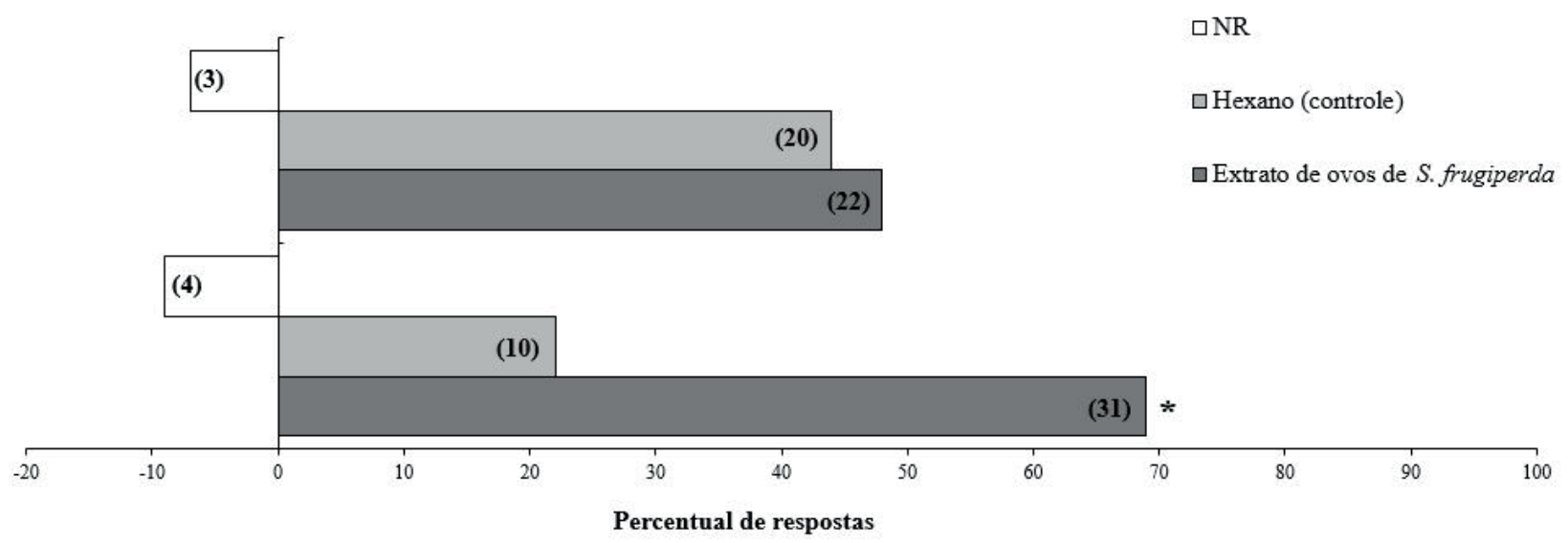

Fig. 1. Percentuais de respostas quimiotáxicas de fêmeas de Trichogramma pretiosum Riley, 1879 (até 24 horas de idade), com e sem experiência a extrato de ovos de Spodoptera frugiperda (J. E. Smith, 1797) testadas e olfatômetro de dupla escolha a extrato de ovos de S. frugiperda e hexano (controle). Números entre parênteses representam a quantidade de insetos responsivos, ou não, aos tratamentos. Valor seguidos de asterisco difere $(p<0,05)$ do tratamento.

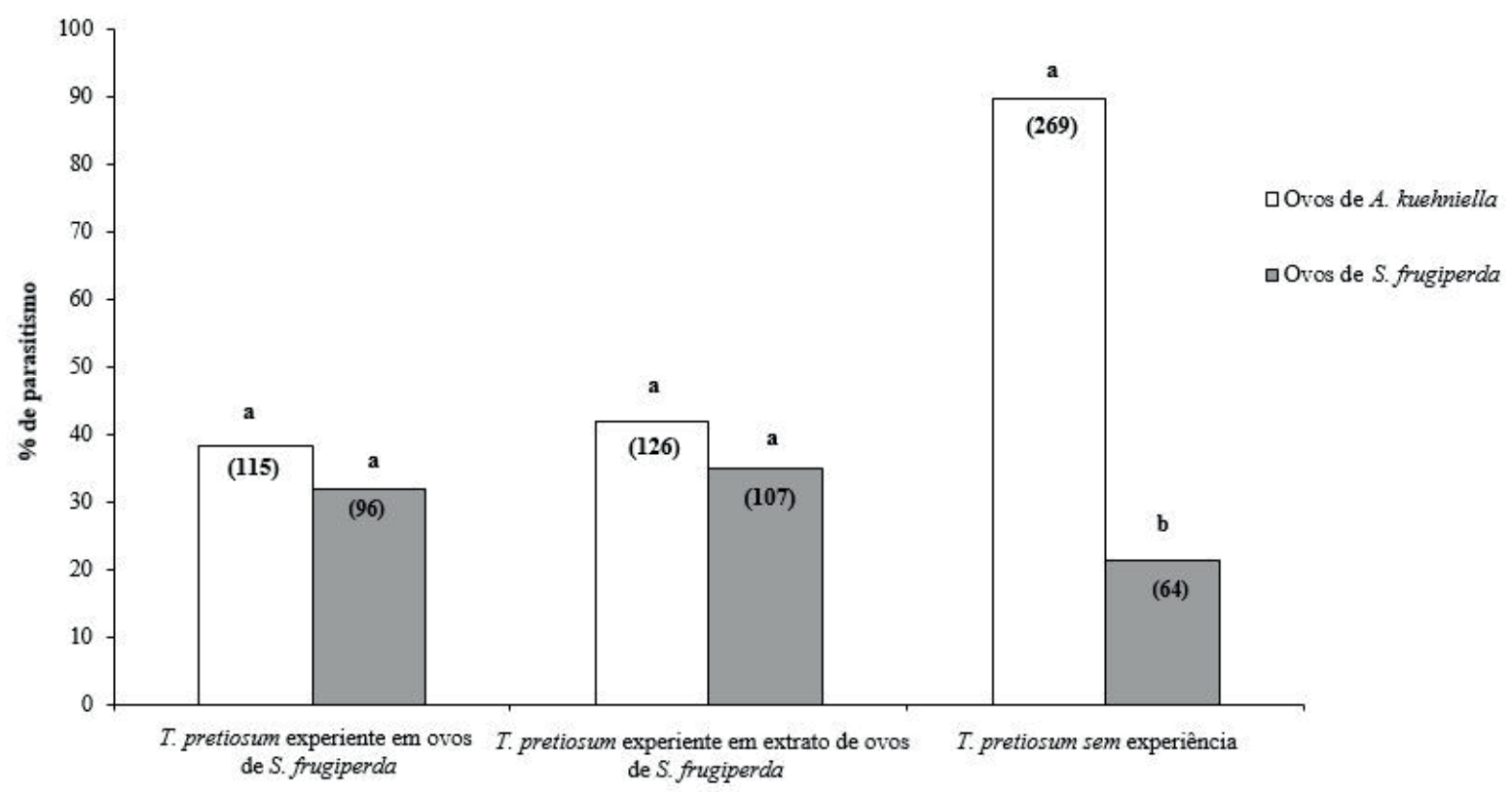

Fig. 2. Percentual de parasitismo de Trichogramma pretiosum Riley, 1879 inexperientes e experientes em ovos ou extratos de ovos de Spodoptera frugiperda (J. E. Smith, 1797) $(\mathrm{n}=30)$ expostos simultaneamente a ovos de Ephestia kuehniella Zeller, 1879 e S. frugiperda. Valores entre parênteses indicam o número de insetos emergidos $(\mathrm{p}<0,05)$.

O maior parasitismo em ovos mais novos também foi observado por Pizzol et al. (2012) os quais verificaram que Trichogramma cacoeciae Marchal, 1927 parasitou mais ovos de Lobesia botrana Denis \& Schiffermuller, 1775 (Lepidoptera: Tortricidae) com 24 e 48 horas de idade em relação aos de 72 e 96 . O mesmo foi constatado para $T$. pretiosum em ovos de Bonagota salubricola Meyrick, 1937 (Lepidoptera: Tortricidae), que preferiram os de até 48 horas (PASTORI et al., 2010). Da mesma forma que os parasitoides anteriores, Trichogramma japonicum Ashmead, 1904, Trichogramma dendrolimi Matsumura, 1926 e Trichogramma chilonis Ishii, 1941 também apresentaram médias de parasitismo mais elevadas em ovos de Chilo suppressalis com dois dias de idade em comparação com os de três e quatro (ZHANG et al., 2014).

O sucesso de parasitismo de T. pretiosum foi de $100 \%$ para todas as idades, indicando altos índices de viabilidade, independentemente da idade do hospedeiro. Segundo JACOB et al. (2006), em algumas espécies ovos mais velhos não apresentam efeito deletério no sucesso de parasitismo. STinguel et al. (2013) também constataram sucesso de parasitismo de $100 \%$ em ovos de Mocis latipes Guenée, 1852 (Lepidoptera: Noctuidae) de 24, 48 e 72 horas, parasitados por T. pretiosum. OliveIRa et al. (2003) registraram 95,5\% de sucesso por Trichogramma maxacalii Voegelé \& Pointel, 1980 em ovos de um dia de idade de Oxydia vesulia Cramer, 1779 (Lepidoptera: Geometridae), em comparação com os de três e cinco dias. 
Assim como registrado neste estudo, um menor tempo de desenvolvimento também for observado para T. pretiosum em ovos mais novos de Helicoverpa zea (RUBERson \& KRING 1993). Miura \& KobaYashi (1998) observaram apenas desenvolvimento de Trichogramma chilonis em ovos de Plutella xylostella de 24 e 48 horas, sendo que nos de 72 h não houve emergência. Embora, a razão sexual possa variar em função de fatores do ambiente, densidade, tipo de hospedeiro e constituição nutricional do ovo (SUZUKI, 1984; VINSON, 1997), neste trabalho, este fator não foi influenciado pela idade do ovo. Este resultado é corroborado por PASTORI et al. (2010), os quais verificaram que a razão sexual da prole de Trichogramma maxacalli não foi afetada em ovos de Bonagota salubricola, com idades entre zero e 72 horas. $\mathrm{O}$ mesmo já havia sido registrado para T. maxacalli, em ovos de Oxydia vesulia, com idades de um, três e cinco dias (Oliveira et al., 2003) e para Trichogramma cacoeciae, em ovos de Lobesia botrana, com idade de zero, 24, 48 e 72 horas de idade (Moreno et al., 2009).

Fêmeas de T. pretiosum adquiriram experiência após 5 horas em contato com ovos de $S$. frugiperda, enquanto que as inexperientes e as expostas por um período mais curto parasitaram menor número de ovos. Da mesma forma, somente as experientes foram atraídas ao extrato de ovos deste hospedeiro. Observou-se também que fêmeas sem experiência parasitaram mais o hospedeiro de origem (Ephestia kuehniella), enquanto que nas experientes, o parasitismo foi similar nas duas espécies.

Em holometábolos, a aquisição de aprendizado está relacionada às alterações do sistema nervoso, que podem ocorrer durante a fase juvenil e persistir na fase adulta (BJORKSTEN \& HOFFMAN, 1998; BARRON \& CORBET, 1999; TogNon et al., 2013, 2014) ou ser desencadeada na fase adulta, após a experiência em novos hospedeiros (BARron, 2001), como observado neste trabalho. Fêmeas de Leptopilina heterotoma Thompson, 1862 (Hymenoptera: Eucoilidae) expostas a larvas do hospedeiro Drosophila subobscura Sturtevant, 1942 (Diptera: Drosophilidae) juntamente com os odores de maçã, cogumelos ou de ambos, também responderam significantemente mais ao odor com o qual tiveram experiência (Vet \& Schoonman, 1988). Em T. pretiosum e T. atopovirilia fêmeas expostas a ovos de $S$. frugiperda também diminuíram o tempo de busca e parasitismo neste hospedeiro (BESERRA \& PARRA, 2003).

Este fato está provavelmente relacionado a alterações no sistema nervoso que podem ocorrer já na fase adulta. Esta hipótese é corroborada pelo trabalho de BIEBER \& FULDNER, (1979), os quais observaram um crescimento de $58 \%$ do protocérebro e $38 \%$ do deutocérebro na fase adulta de Aleochara curtula Goeze, 1777 (Coleoptera: Staphylinidae) sem a presença de estímulos aparentes. Fato semelhante foi constatado em adultos de Drosophila melanogaster Meigen, 1830 (Diptera: Drosophilidae), com um crescimento de cerca de $15 \%$ do número total de fibras do corpo cogumelar nos primeiros sete dias de vida deste inseto (Technau, 1984). Segundo o autor, as modificações estão relacionadas a três fatores: a) a idade do inseto, sendo que o incremento de fibras é observado nas primeiras duas semanas de vida e decresce a partir terceira semana; b) ao sexo, fêmeas apresentam maior desenvolvimento de fibras comparadas aos machos e c) a percepção de estímulos ambientais, adquiridos principalmente no momento do voo, associados à visão e ao olfato.

Neste estudo o aprendizado de fêmeas de T. pretiosum ocorreu tanto pela exposição destas aos ovos, como ao extrato de ovos do hospedeiro. Esta experiência provavelmente foi obtida a partir de sensilas localizadas nas antenas e/ ou no ovipositor as quais, segundo LE REC \& WAJNBERG (1990), são estimuladas por compostos presentes sobre ou dentro do córion do hospedeiro (VINSON 1998; NURINDAH \& GoRDH, 1999). Indivíduos de T. pretiosum criados em ovos de Ephestia kuehniella, também foram capazes de reconhecer e parasitar significativamente mais ovos de Anticarsia gemmatalis, Pseudoplusia includens Walker, 1857 (Lepidoptera: Noctuidae), Diatraea saccharalis Fabricius 1794 (Lepidoptera: Crambidae) e $S$. frugiperda após experiência com cada um dos ovos destas espécies, por 24 horas (Siqueira et al., 2011). Segundo Steidle \& VAN LOON (2002), esta é uma estratégia usada para lidar com a grande variabilidade de informações químicas presente no ambiente.

A experiência de um parasitoide está associada à aquisição de informações e estímulos os quais podem conferir vantagens adaptativas a este, aumentando sua performance reprodutiva e de busca por hospedeiros (WAJNBERG \& Colazza, 2012). A aprendizagem também aumenta as chances de sobrevivência, pois nem sempre seu hospedeiro de origem se encontra disponível no ambiente (STRAND \& VINSON, 1983).

Em um contexto aplicado, a principal vantagem do aprendizado na fase adulta está relacionada à possibilidade de manipular o comportamento de busca do parasitoide quando expostos aos voláteis do hospedeiro ao qual o controle se destina. Vet \& Groenewold (1990) já haviam apontado esta como a forma promissora de aplicação da aprendizagem no manejo de pragas, fato corroborado por MEINERs \& PERI (2013).

A partir dos resultados obtidos observou-se que $T$. pretiosum apresenta preferência por hospedeiros com até 24 horas de idade. A espécie possui capacidade de aprender em fase adulta, após experiência prévia a ovos ou extrato do hospedeiro, por no mínimo 5 horas. Fêmeas experientes necessitam de um menor tempo de exposição a ovos de $S$. frugiperda, quando comparados a fêmeas inexperientes. O aprendizado de T. pretiosum a extrato e ovos de S. frugiperda indica que é possível modular a percepção química deste, direcionando o parasitoide para determinados hospedeiros. No entanto, são necessários estudos que avaliem esta hipótese em condições de campo, onde existe uma maior variedade de estímulos.

Agradecimentos. À Coordenação de Aperfeiçoamento de Pessoal de Nível Superior (CAPES) e ao Conselho Nacional de Desenvolvimento Científico e Tecnológico (CNPq) pelas bolsas concedidas aos autores. 


\section{REFERÊNCIAS BIBLIOGRÁFICAS}

Almeida, G. D.; Zanuncio, J. C.; Pratissoli, D.; Andrade, G. S.; Cecon, P. R. \& SERrÃo, J. E. 2010. Effect of azadirachtin on the control of Anticarcia gemmatalis and its impact on Trichogramma pretiosum. Phytoparasitica 38:413-419.

Ayres, M.; Ayres JúnIOr, M.; Ayres, D. L. \& SAntos, A. A. 2007. BioEstat 5.0 - Aplicações Estatísticas nas Áreas das Ciências Biológicas e Médicas. Belém, Sociedade Civil Mamirauá. 364p.

Barron, A. B. 2001. The life and Death of Hopkins Host-Selection Principle. Journal of Insect Behavior 14(6):725-737.

Barron, A. B. \& Corbet, S. A. 1999. Preimaginal conditioning in Drosophila revisited. Animal Behaviour 58(1):621-628.

Beserra, E. B. \& PArra, J. R. P. 2003. Comportamento de parasitismo de Trichogramma atopovirilia Oatman \& Platner e Trichogramma pretiosum Riley (Hynmenoptera, Trichogrammatidae) em posturas de Spodoptera frugiperda (J. E. Smith) (Lepidoptera, Noctuidae). Revista Brasileira de Entomologia 47(2):205-209.

Beserra, E. B. \& PARRA, J. R. P. 2005. Impact of the number of Spodoptera frugiperda egg layers on parasitism by Trichogramma atopovirilia. Scientia Agricola 62(2):190-193.

Bieber, M. \& Fuldner, D. 1979. Brain growth during the adult stage of a holometabolos insect. Naturwissenshaften 66:426.

Bjorksten, T. A. \& Hoffmann, A. A. 1998. Plant cues influencing searching behavior and parasitism in the egg parasitoid Trichogramma nr. Brassicae. Ecological Entomology 23(4):355-362.

Carneiro, T. R.; Fernandes, O. A. \& Cruz, I. 2006. Resposta olfativa de Telenomus remus Nixon (Hymenoptera; Scelionidae) a voláteis emitidos por Spodoptera frugiperda (J. E. Smith) (Lepidoptera: Noctuidae). Entomotropical 21(3):153-159.

Davies, A. P.; Carr, M. C.; Scholz, B. C. G. \& Zalucki, M. P. 2011. Using Trichogramma Westwood (Hymenoptera: Trichogrammatidae) for insect pest biological control in cotton crops: an Australian perspective. Austral Entomology 50(4):424-440.

Figueiredo, M. L.C.; Cruz, I.; Silva, R. B. \& Foster, J. E. 2015. Biological control with Trichogramma pretiosum increases organic maize productivity by $19.4 \%$. Agronomy for Susteinable Development 35(3):1175-1183.

Gazit, Y.; Lewis, J. \& Tumlinson, J. H. 1996. Arrestment of Telenomus remus (Hymenoptera: Scelionidae) by a kairomone associated with eggs of its host, Spodoptera frugiperda (Lepidoptera: Noctuidae). Biological Control 6:283-290

Goulart, M. M.; Bueno, A. F.; Bueno, R. C. O. \& Diniz, A. F. 2011. Host preference of the egg parasitoids Telenomus remus and Trichogramma pretiosum in laboratory. Revista Brasileira de Entomologia 55(1):129133.

Hopkins, A. D. 1917. A discussion of H. G. Hewitt's paper on insect behavior. Journal of Economic Entomology 10:92-93

JACOB, H. S.; Joder, A. \& BATChELOR, K. L. 2006. Biology of Stethynium sp. (Hymenoptera: Mymaridae), a native parasitoid of an introduced weed biological control agent. Environmental Entomology 35:630-636.

Le Rec, A. \& WajnberG, E. 1990. Sensory receptors of the ovipositor of Trichogramma maidis (Hym. Trichogrammatidae). Entomophaga 35:293-299.

Meiners, T. \& Peri, E. 2013. Chemical ecology of insect parasitoids: essential elements for developing effective biological control programmes. In: Wajnberg, E. \& Colazza, S. eds. Chemical Ecology of Insects Parasitoids. Chichester, Wiley-Blackwell. 312p.

MiURA, K. \& KoBAYASHI, M. 1998. Effects of host-eggs age on the parasitismo by Trichogramma chiloniis Ishii (Hymenoptera: Trichogrammatidae), an egg parasitoid of the diamondback moth. Applied Entomology and Zoology 33(2):219-222.

Moreno, F.; Moreno-Pérez, I. \& Marco, V. 2009. Effects of Lobesia botrana (Lepidoptera: Tortricidae) egg age, density, and UV treatment on parasitismo and development of Trichogramma cacoeciae (Hymenoptera: Trichogrammatidae). Physilogical Ecology 38(5):15131520 .

Nurindah, B. W. C. \& Gordh, G. 1999. Experience acquition by Trichogramma australicum Girault (Hymenoptera: Trichogrammatidae). Australian Journal of Entomology 38:15-119.
Oliveira, H. N.; Pratissoli, D.; Zanuncio, J. C. \& Serrão, J. E. 2003. Influência da idade dos ovos de Oxydia vesulia no parasitismo de Trichogramma maxacalli. Pesquisa Agropecuária Brasileira 48(4):551-554.

Pak, G. A.; Buis, H. C. E. M.; Heck, I. C. C. \& Hermans, M. L. G. 1986. Behavioural variations among strains of Trichogramma spp.: host-age selection. Entomologia Experimentalis et Applicata 40:247-258.

PARrA, J. R. P. 1997. Técnicas de criação de Anagasta kuehniella, hospedeiro alternativo para a produção de Trichogramma. In: PARRA, J. R. P. \& ZuCCHI, R. A. eds. Trichogramma e o controle biológico aplicado. Piracicaba, FEALQ, p.121-150.

PARrA, J. R. P. 2001. Técnicas de criação de insetos para programas de controle biológico. Piracicaba, FEALQ. 137p.

Pastori, P. L.; Monteiro, L. B.; Botton, M. \& Pratissoli, L. 2010. Efeito da idade do parasitoide e do hospedeiro na reprodução de Trichogramma pretiosum (Hymenoptera: Trichogrammatidae) em ovos de Bonagota salubricola (Myrick) (Lepidoptera: Tortricidae). Arquivos do Instituto Biológico 77(2):349-353.

Pizzol, J.; Desneux, N.; Wajnberg, E. \& Thiéry, D. 2012. Parasitoid and host egg ages have independent impact on various biological traits in a Trichogramma species. Journal of Pest Science 85:489-496.

Querino, R. B.; SiLvA, N. N. P. \& ZuCCHI, R. A. 2016. Natural parasitism by Trichogramma spp. in agroecosystems of the Mid-North, Brazil. Ciência Rural 46(9):1521-1523.

Ruberson, J. R. \& Kring, T. 1993. Parasitism of developing eggs by Trichogramma pretiosum (Hymenoptera: Trichogrammatidae): host age preference and suitability. Biological Control 3:39-46.

Schmidt, J. M. \& Sмiтh, J. J. B. 1988. Host volume measurement by Trichogramma mechanism and application to biological control. In: International Symposium on Trichogramma and other egg Parasitoids. Paris, INRA, p. 239-248.

Siqueira, J. R.; Bueno, R. C. O. De F.; Bueno, A. F. \& Vieira, S. S. 2011. Preferência hospedeira do parasitoide de ovos Trichogramma pretiosum. Ciência Rural 42(1):1-5.

Steidle, J. L. M. \& VAN LoOn, J. J. A. 2002. Chemoecology of parasitoid and predator oviposition behavior. In: Hilker, M. \& MeInERs, T. eds. Chemoecology of Insect Eggs and Eggs Deposition. Berlin, Blackwell Publishing, p. 291-317.

Stinguel, P.; Carvalho, J. R.; Pratissoli, D.; Zuim, V. \& Mardgan, L. 2013. Efeito da idade dos ovos de Mocis latipes (Lepidoptera: Noctuidae) sobre o parasitismo de Trichogramma pretiosum (Hymenoptera: Trichogrammatidae) com diferentes idades. Nucleus 10(2):265-274.

Strand, M. R. \& Vinson, S. B. 1983. Analysis of an egg recognition kairomone of Telenomus heliothisdis (Hymenoptera: Scelionidae). Isolation and host function. Journal of Chemical Education 9:423-432.

Strand, M. R. \& Vinson, S. B. 1986. Host acceptance behavior of Telenomus heliothidis (Hymenoptera: Scelionidae) toward Heliothis virescens (Lepidoptera: Noctuidae). Annals of the Entomological Society of America 76(4):781-785.

Sujit, E. R.; Costa, M. L.; Pires, C. S. S.; Colazza, S. \& Borges, M. 2002. Inter and intra-guild interactions in egg parasitoid species of the sybean stink bus complex. Pesquisa Agropecuária Brasileira 37(11):1541-1549.

Suzuki, Y.; Tsuji, H. \& SASAKaWA, M. 1984. Sex allocation and effects of superparasitism on secondary sex ratios in the gregarious parasitoid, Trichogramma chilonis (Hymenoptera: Trichogrammatidae). Animal Behavior 32:478-484.

Technau, G. M. 1984. Fiber number in the mushroom bodies of adult Drosophila melanogaster depends on age, sex and experience. Journal Neurogenetics 21:183-196.

TognON, R.; SANT'ANA. J. \& JAHNKE. S. M. 2014. Influence of original host on chemotaxic behaviour and parasitismo in Telenomus podisi Asmead (Hymenoptera: Platygastridae). Bulletin of Entomological Research 104:781-787.

Tognon, R.; SANT’AnA. J. \& JAHnKe, S. M. 2013. Aprendizagem e memória de Telenomus podisi (Hymenoptera, Platygastride). Iheringia, Série Zoologia 103(3):266-271

Vet, L. E. M. \& Schoolman, G. 1988. The influence of previous foraging experience on microhabitat acceptance in Leptopilina heterotoma. Journal of Insect Behavior 1(4):387-392. 
Vet, L. M. E. \& Groenewold, A. W. 1990. Semiochemicals and learning in parasitoids. Journal of Chemical Ecology 16(11):3119-3135.

Vinson, S. B. 1976. Host selection by insect parasitoids. Annual Review of Entomology 21:109-133.

Vinson, S. B. 1998. The general host selection behavior of parasitoid Hymenoptera and a comparison of initial strategies utilized by larvaphagous and oophagous species. Biological Control 11(2):79-96.

Vinson, S. B. 1997. Comportamento de seleção hospedeira de parasitoides de ovos, com ênfase na família Trichogrammatidae. In: PARRA, J. R. P. \& ZUCCHI, R. A. Trichogramma e o controle biológico aplicado. Piracicaba, Fealq, p. 67-210.

Volpe, H. X. L.; Bortoli, R. T.; Thuler, C. L. T. P.; Viana, C. L. T. P. \& Goulart, R. M. 2006. Avaliação de características biológicas de
Trichogramma pretiosum Riley (Hymenopetra: Trichogrammatidae) criado em três hospedeiros. Arquivos do Instituto Biológico 73(3):311315.

WajnberG, E. \& Colazza, S. 2012. Chemical Ecology of Insect Parasitoids. Chichester, Wiley-Blackwell. 312p.

Zhang, J. J.; Ren, B. Z.; Yuan, X. H.; Zang, L. S.; Ruan, C. C.; Sun, G. \& ShaO, X. 2014. Effects of host-egg ages on host selection and suitability of four Chinese Trichogramma species, egg parasitoids of the rice striped stem borer, Chilo suppressalis. BioControl 59(2):159-166.

Zuim, V.; Paes, J. P. P.; Carvalho, J. R.; Stinguel, P. \& Pratissoli, D. 2013. Parasitismo de Trichogramma exiguum: influência do desenvolvimento embrionário dos ovos e da idade do parasitoide. Revista Verde 8(1):211 217. 